\title{
Probing nonorthogonality of eigenfunctions and its impact on transport through open systems
}

\author{
Matthieu Davy ${ }^{1, *}$ and Azriel Z. Genack ${ }^{2}$ \\ ${ }^{1}$ Univ Rennes, CNRS, Institut d'Electronique et de Télécommunications de Rennes (IETR) - UMR 6164, F35000 Rennes, France \\ ${ }^{2}$ Department of Physics, Queens College and Graduate Center of the City University of New York, Flushing, New York 11367, USA
}

(Received 3 May 2019; revised manuscript received 10 September 2019; published 16 October 2019)

\begin{abstract}
The degree of nonorthogonality of eigenstates of non-Hermitian systems governs nuclear scattering, electronic conductance, and wave propagation in disordered media. Here, we determine the impact of non-Hermiticity upon eigenstates inside an open random cavity from measurements of the modal overlap matrix of transmitted microwave radiation. Increasing eigenfunction correlation with spectral modal overlap brought about by the openness of the system leads to modal transmission greatly exceeding unity accompanied by strong modal anticorrelation guaranteeing that transmission does not exceed unity.
\end{abstract}

DOI: 10.1103/PhysRevResearch.1.033026

\section{INTRODUCTION}

Excitation of and transport through a complex medium reflect the character of the eigenstates of the wave equation. In quantum systems, these are referred to as energy levels, while for classical waves, these are called quasinormal modes, or simply modes. The real and imaginary parts of the eigenvalues of the wave equation are the central frequencies and decay rates of the modes and the eigenstates give the corresponding spatial structure over the volume. Though it is generally not possible to solve for the eigenvalues of the Hamiltonian in large complex systems, the resonances correspond to the poles of the scattering matrix and can be extracted from measurement of the spectrum of its elements [1-7].

A single mode may be excited when the sample is illuminated on resonance with a spectrally isolated mode. However, as the coupling of the sample to the exterior and internal dissipation increase, modes broaden and overlap spectrally. In such non-Hermitian systems, the eigenfunctions are no longer orthogonal [1,5,8-12]. The non-Hermiticity of open systems is observed in the overlapping nuclear resonance in neutron scattering and used to interpret the statistics of width and spacing of nuclear levels [13-17]. The degree of modal overlap also determines the statistics of electronic conductance $[18,19]$ as well as classical wave transmission and level statistics in random media and chaotic cavities [20-22], but these statistics alone do not directly determine scattering spectra. This would require knowledge of the statistics of correlation and the degree of excitation of the eigenfunctions. However, directly probing the generic statistics of the eigenvectors of non-Hermitian systems has remained a challenge.

The nonorthogonality of eigenfunctions is crucial for the viability in quantum computing [23]. In photonic systems,

\footnotetext{
*Corresponding author: matthieu.davy@univ-rennes1.fr

Published by the American Physical Society under the terms of the Creative Commons Attribution 4.0 International license. Further distribution of this work must maintain attribution to the author(s) and the published article's title, journal citation, and DOI.
}

it leads to the existence of exceptional points in systems that incorporate both gain and loss but in which parity-time symmetry is preserved [24,25] and to the enhancement of the linewidth and spontaneous emission rates in laser resonators [26-28]. Its impact has also been explored in localized plasmonic surfaces [29], dielectric microcavities [30,31] and chaotic systems with small perturbations [32,33]. Thus the impact of non-Hermiticity on wave functions is critical to the deposition and evolution of energy in open linear and nonlinear systems, communications, imaging, spectral analysis, and resource exploration.

In this paper, we explore the consequences of nonHermiticity and consequent modal overlap in an open random microwave cavity. We demonstrate the correlation of modal components of the transmission matrix (TM) in nonHermitian systems and their relation to the correlation of eigenfunctions over the entire volume of the sample. Using a modal analysis of the TM in ensembles of disordered systems, we show that off-diagonal elements of the transmission overlap matrix are negative and exhibit universal scaling in agreement with predictions of random matrix theory $[10,12,34]$. This is associated with enhanced modal excitation, with an average value in random media that approaches the dimensionless conductance, and can greatly exceed unity. The linkage of enhanced modal transmission and destructive interference among correlated speckle patterns of spectrally overlapping resonances ensures that net transmission does not exceed unity.

\section{NONORTHOGONALITY OF EIGENFUNCTIONS}

The quasinormal modes of a system are the eigenfunctions $\phi_{n}(r)$ of the wave equation $\Delta \phi_{n}(r)=-\epsilon(r) \tilde{\omega}_{n}^{2} / c_{0}^{2} \phi_{n}(r)$ that satisfy outgoing boundary conditions $[1,5,35] . c_{0}$ is the speed of light and the disorder relies on the spatial distribution of the permittivity $\epsilon(r)$. The resonances are the complex eigenvalues $\tilde{\omega}_{n}=\omega_{n}-i \Gamma_{n} / 2$, where $\omega_{n}$ is the central frequency and $\Gamma_{n}$ is the linewidth. The eigenfunctions are normalized to form a complete biorthogonal set so that $\phi_{n}^{T} \phi_{m}=\delta_{n m}$. 
The overlap matrix $O_{m n} \equiv\left(\phi_{m}^{\dagger} \phi_{n}\right)^{2}=\left[\int d r \phi_{m}^{*}(r) \phi_{n}(r)\right]^{2}$ characterizes the correlation between eigenvectors of the wave equation over the volume $[10,12,34]$. The diagonal elements of $O_{m n}$ are equal to the Petermann factor, $K_{n} \equiv O_{n n}$, which is a measure of the degree of complexness of the eigenfunctions [36-40]. The Petermann factor characterizes the excess spontaneous emission for laser cavities and governs the linewidth of lasing modes [26-28,41]. For small modal overlap, the eigenfunctions nearly coincide with the real eigenfunctions of the closed system so that $K_{n} \sim 1$. However, $K_{n}$ increases as the coupling of the sample to its surroundings increases [37] and can have values exceeding 1000 [27]. The off-diagonal elements of $O$ give the degree of correlation between eigenfunctions. Since the eigenfunctions are complete, $\Sigma_{m} O_{n m}=1$ [10], the enhancement of $K_{n}$ with increasing modal overlap implies nonvanishing and negative-on-average correlation $O_{n \neq m}<0$ for overlapping eigenfunctions, in contrast to $O_{n \neq m}=0$ in Hermitian systems.

Measuring the overlap matrix would require a noninvasive scan of the spatial profile of the eigenfunctions inside the sample, which is not possible in most cases. We show, however, that the measurement of the spectrum of the TM makes it possible to compute a transmission overlap matrix whose statistics reflects those of the overlap matrix. The expansion of the scattering matrix in terms of quasinormal modes is $S=I-i W^{\mathrm{T}}[\omega-\tilde{\Omega}]^{-1} W[5,9]$. The matrix $W$ of vectors $W_{n}$ is the projection of eigenfunctions $\phi_{n}$ onto the channels of the sample and $\tilde{\Omega}$ is the diagonal matrix of eigenvalues $\tilde{\omega}_{n}$. The TM can then be expressed as a superposition of modal TMs (MTMs) [42]:

$$
t(\omega)=-i \Sigma_{n} \frac{W_{R n} W_{L n}^{\mathrm{T}}}{\omega-\tilde{\omega}_{n}} .
$$

$W_{L n}$ and $W_{R n}$ are the components of the $W_{n}$ vector associated with the left and right sides of the sample, respectively.

The statistics of the modes in chaotic cavities and random systems are accurately modeled within the effective Hamiltonian formalism. The eigenfunctions correspond to the eigenvectors of the effective Hamiltonian $H_{\text {eff }}=H_{0}-\frac{i}{2} V V^{T}$, where $H_{0}$ is the real Hermitian Hamiltonian of the closed system and the real matrix $V$ describes the coupling of the system through channels. We demonstrate in the Supplemental Material (SM) [43] that the correlation of eigenfunctions is related to the correlation of their projections onto the coupling channels as $[5,16,43]$

$$
O_{m n}=-\frac{\left(W_{m}^{\dagger} W_{n}\right)^{2}}{\left(\tilde{\omega}_{m}-\tilde{\omega}_{n}^{*}\right)^{2}} .
$$

For $m=n$, Eq. (2) gives the relation between the mode linewidths and the coupling vectors, $K_{n}=\left\|W_{n}\right\|^{4} / \Gamma_{n}^{2}[16]$. Because we measure the TM rather than the scattering matrix, the relative phase and magnitudes of the vectors $W_{L n}$ and $W_{R n}$ are unknown; only the MTMs $t_{n}=-i W_{R n} W_{L n}^{\mathrm{T}}$ can be extracted. We therefore define the "transmission overlap matrix":

$$
\tilde{O}_{m n}=-\frac{4\left(W_{R m}^{\dagger} W_{R n}\right)\left(W_{L m}^{\dagger} W_{L n}\right)}{\left(\tilde{\omega}_{m}-\tilde{\omega}_{n}^{*}\right)^{2}}=-\frac{4 \operatorname{Tr}\left(t_{m}^{\dagger} t_{n}\right)}{\left(\tilde{\omega}_{m}-\tilde{\omega}_{n}^{*}\right)^{2}} .
$$

The diagonal elements gives the modal strengths in transmission $\tilde{O}_{n n}=T_{n}=\left\|W_{R n}\right\|^{2}\left\|W_{L n}\right\|^{2} /\left(\Gamma_{n} / 2\right)^{2}$. $\tilde{O}\left(\tilde{\omega}_{m}, \tilde{\omega}_{n}\right)$ and (a)

INCOMINC

CHANNELS

OUTGOING

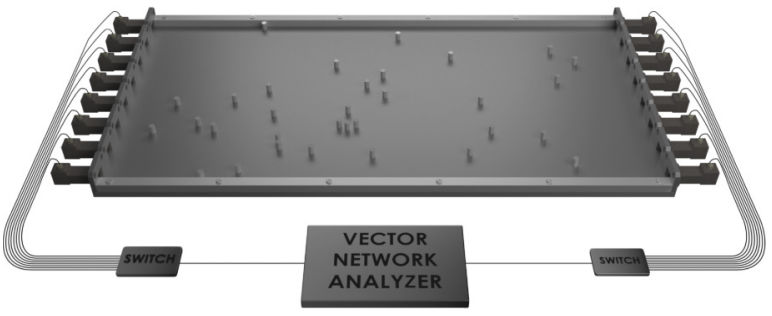

(b)

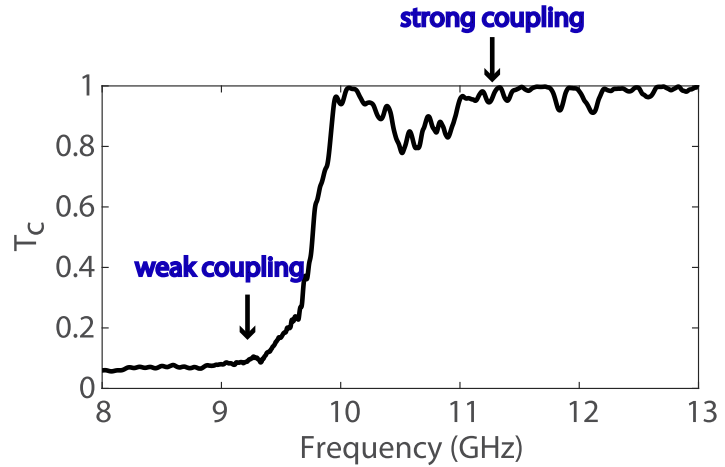

FIG. 1. (a) Experimental setup. (b) Transmission through the antennas as a function of frequency. The arrows indicate the frequency ranges corresponding to weak and strong coupling of the antennas.

$O\left(\tilde{\omega}_{m}, \tilde{\omega}_{n}\right)$ are equal for extended states in the limit $N \gg 1$ for which $\left\|W_{R n}\right\| \sim\left\|W_{L n}\right\|[43]$.

We measure the TM of a multichannel random system [see Fig. 1(a)]. The disordered aluminum cavity of height $H=8 \mathrm{~mm}$, width $W=250 \mathrm{~mm}$, and length $L=500 \mathrm{~mm}$ supports a single transverse mode in the vertical direction so the system is essentially two dimensional. The randomly positioned scattering elements are 6-mm-diameter aluminum cylinders. The TM which is the part of the scattering matrix associated with the transmission coefficients between the left and right sides of the sample, respectively, is measured in the microwave range between two linear arrays of $N=8$ antennas that are coaxial to waveguide adapters [42]. Spectra of each transmission coefficients of the TM are obtained sequentially using two electromechanical switches and the two ports of a vector network analyzer. The openings of the system are fully controlled by the antennas [44] but the interface may still be strongly reflective due to the metallic region surrounding each coupler. Figure 1(b) shows the spectrum of the emission from the antennas determined from $T_{c}(\omega)=1-\left|\left\langle S_{c c}(\omega)\right\rangle\right|^{2}$, where $\left\langle S_{c c}(\omega)\right\rangle$ is the mean reflection parameter at each antenna.

The degree of modal overlap in random media depends upon the degree of disorder and the openness of the sample boundaries. The average modal overlap may be characterized by the Thouless number $\delta$, which is the ratio of the average linewidth to the average level spacing $\delta=\left\langle\Gamma_{n}\right\rangle / \Delta \omega$. $\delta$ reflects the degree of spatial localization since tightly localized modes couple weakly to the surroundings through the sample boundaries and so have narrow linewidths $[21,45]$. We carry out measurements in three ensembles with moderate modal overlap in frequency ranges in which (1) the antennas are 


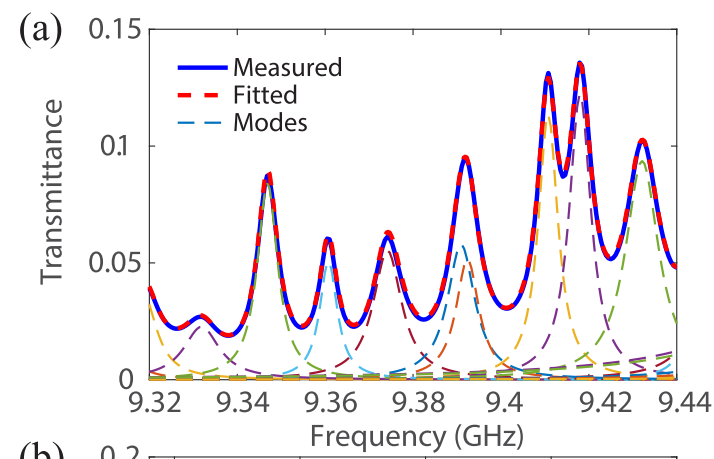

(b)

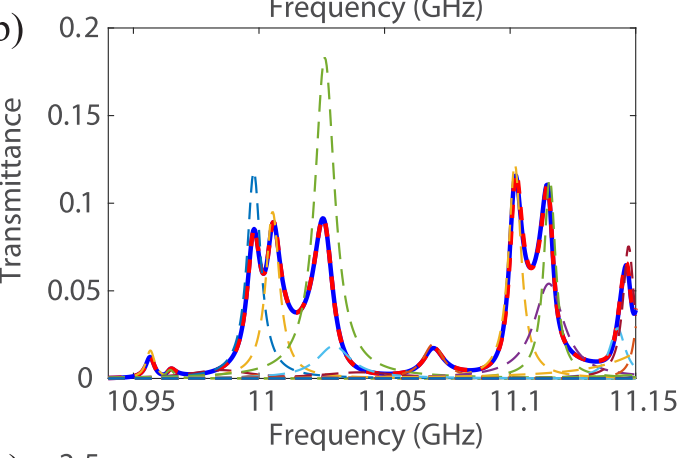

(d)

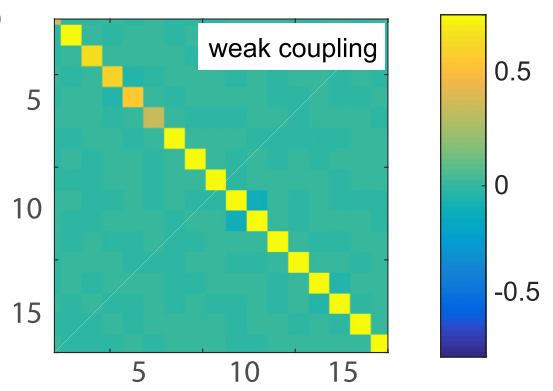

(e)

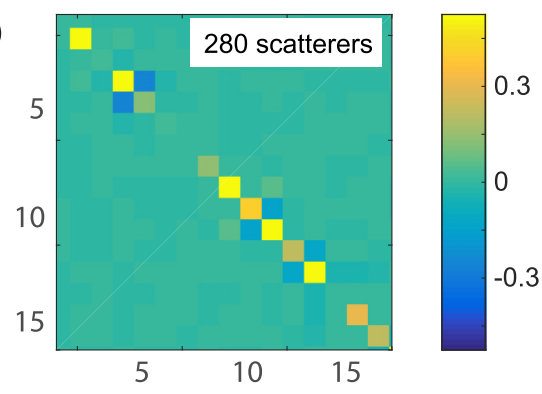

(c)

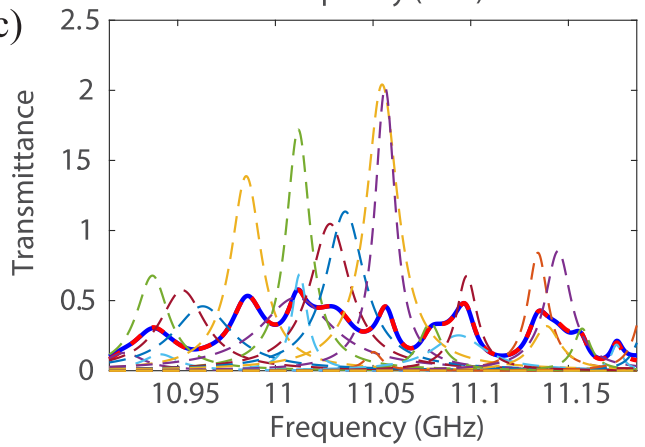

(f)

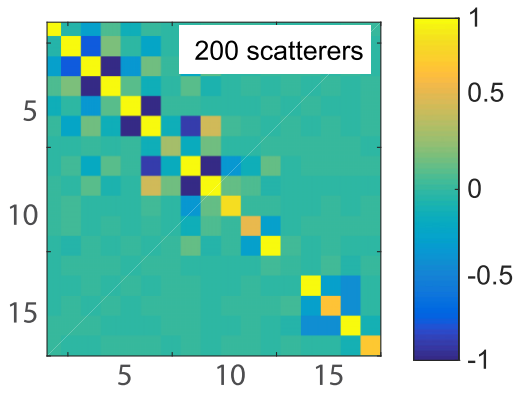

FIG. 2. (a)-(c) Measured transmittance $T(\omega)=\Sigma_{a, b}\left|t_{b a}(\omega)\right|^{2}$ and the underlying modal structure for (a) weak coupling to a sample with 30 cylinders and (b) for strong coupling to a sample with 250 cylinders and (c) 200 cylinders. (d)-(f) Real part of the overlap matrix for 17 modes of the ensembles corresponding to spectra shown in (a)-(c). The modal strength and negative correlation between neighboring modes are seen in the increase of the diagonal and off-diagonal terms, respectively, with increasing modal overlap from (d) to (f).

weakly coupled to a sample ( $f$ between 9.3 and $9.5 \mathrm{GHz}$ and $\left.T_{c} \sim 0.09\right)$ with 30 cylinders contained within a cavity, for which $\delta \sim 0.5$; and $(2,3)$ the antennas are strongly coupled to a sample ( $f$ between 10.8 and $11.5 \mathrm{GHz}$ and $T_{c} \sim 0.98$ ) and the disorder is strong. For the samples with 280 and 200 scatterers, $\delta \sim 1.2$ and $\delta \sim 2.01$, respectively.

Spectra of the transmittance $T(\omega)=\Sigma_{a b}\left|t_{b a}(\omega)\right|^{2}$, which is the sum over flux transmission coefficients between all incoming and outgoing channels, and the modal strengths in transmission $T_{n}(\omega)=\left\|W_{R n}\right\|^{2}\left\|W_{L n}\right\|^{2} /\left|\omega-\tilde{\omega}_{n}\right|^{2}$, are shown in Figs. 2(a)-2(c). For classical waves, the average transmittance corresponds to the dimensionless conductance, $g=$ $\langle T(\omega)\rangle[18-21,45,46]$. We observe in Fig. 2(a) that the conductance $g \sim 0.06$ is small when the antennas are weakly coupled to the system as a result of the small modal overlap and absorption over the sample, but $g$ increases with increasing coupling of the antennas to the sample. The resonances $\tilde{\omega}_{n}$ and the vectors $W_{L n}$ and $W_{R n}$ are extracted from spectra of transmission coefficients using the harmonic inversion technique $[2,42]$. In the vicinity of the central frequency of an isolated mode, peaks in transmission spectra correspond to resonances with individual modes, $T(\omega) \sim T_{n}(\omega)$. But as modal overlap increases, the strength of modes on resonance $T_{n}=T_{n}\left(\omega_{n}\right)$ is enhanced and may exceed unity.

We compensate for the impact for absorption in order to compute the transmission overlap matrix $\tilde{O}$. Since the linewidths $\Gamma_{n}$ are broadened by absorption, we replace in the denominator of Eq. (3) $\tilde{\omega}_{n}-\tilde{\omega}_{m}^{*}$ by $\tilde{\omega}_{n}-\tilde{\omega}_{m}^{*}+i \Gamma_{a}$, where $\Gamma_{a}$ is the homogeneous absorption rate. To estimate the linewidth $\Gamma_{a}$ associated with homogeneous absorption and losses through the antennas $\tilde{\Gamma}_{n}$, related by $\Gamma_{n}=\tilde{\Gamma}_{n}+\Gamma_{a}$, we observe that the average $\left\langle\tilde{\Gamma}_{n}\right\rangle$ scales linearly with the number of coupled antennas, $\left\langle\tilde{\Gamma}_{n}\right\rangle=2 N\left\langle\tilde{\Gamma}_{n}^{0}\right\rangle$, where $\left\langle\tilde{\Gamma}_{n}^{0}\right\rangle$ is the average linewidth associated to a single antenna coupled to the sample. By disconnecting the antennas from the switches, it is possible to decrease the number of coupled channels and thereby obtain an estimate for $\Gamma_{a}$. In the weak coupling regime, we find $\left\langle\tilde{\Gamma}_{n}^{0}\right\rangle \sim 0.15 \mathrm{MHz}$ and $\Gamma_{a} \sim 3.6 \mathrm{MHz}$, so that the broadening of the resonances in the weak coupling regime is therefore mainly due to homogeneous absorption within the sample. In the strong coupling regime associated to a higher frequency range, $\left\langle\tilde{\Gamma}_{n}^{0}\right\rangle \sim 1 \mathrm{MHz}$ and $\Gamma_{a} \sim 4 \mathrm{MHz}$ show that 


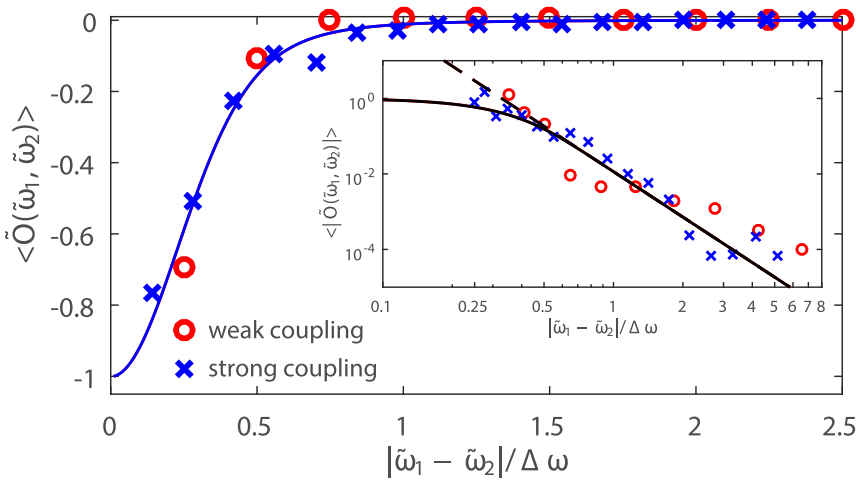

FIG. 3. Measured correlator $\left\langle\tilde{O}\left(\tilde{\omega}_{1}, \tilde{\omega}_{2}\right)\right\rangle$ plotted as a function of $|\delta \tilde{\omega}|=\left|\tilde{\omega}_{1}-\tilde{\omega}_{2}\right| / \Delta \omega$, with weakly (red circles) and strongly (blue crosses) coupled antennas. The lines are fits to theoretical predictions given in Eq. (4). The results are normalized so that $\left\langle\tilde{O}\left(\tilde{\omega}_{1}, \tilde{\omega}_{2}\right)\right\rangle \rightarrow$ -1 as $|\delta \tilde{\omega}| \rightarrow 0$. Inset: $\left|\left\langle\tilde{O}\left(\tilde{\omega}_{1}, \tilde{\omega}_{2}\right)\right\rangle\right|$ in logarithmic scale is seen to scale as $|\delta \tilde{\omega}|^{-4}$ (dashed lines) for $|\delta \tilde{\omega}|>\Delta \omega$.

losses are dominated by the coupling through the antennas for $N=8$.

The real parts of $\tilde{O}\left(\tilde{\omega}_{1}, \tilde{\omega}_{2}\right)$ are shown in Figs. 2(d)-2(f) for the three samples studied. When the antennas are weakly coupled, the overlap matrix $\tilde{O}$ is seen to be close to diagonal as it would be for a closed system. Uniform losses that broaden the linewidths do not alter the orthogonality of eigenfunctions. For the case of strong coupling and $\delta \sim 1.2$, the transmission overlap matrix is also mostly diagonal. However, when two resonances overlap, enhanced diagonal and negative offdiagonal elements are observed, as seen, for instance, for the two resonances with central frequencies around $11.03 \mathrm{GHz}$. For the sample with the smaller number of scatterers and hence shorter mode lifetime and larger mode linewidth, $\delta \sim$ 2.01 , the diagonal part for most modes increases while the off-diagonal terms become more negative.

To carry out measurements on a random ensemble, an ensemble of independent realizations of the cavity is obtained by moving a $10-\mathrm{mm}$-diameter magnet along a line within the medium in steps of $\lambda / 2=12.5 \mathrm{~mm}$, where $\lambda$ is the wavelength at $12 \mathrm{GHz}$. The magnet within the sample is moved by the force of a second magnet above the top plate of the cavity. We find resonances and associated modal coefficients for more than 1000 modes in 40 realizations of two ensembles: (1) weakly coupled antennas with 30 scatterers giving $\delta=$ 0.5 , and (2) strongly coupled antennas with 280 scatterers. Since no scatterers are positioned along the line of motion of the magnet, $\delta$ for this ensemble is increased to $\delta=1.52$ from $\delta=1.2$, for the sample where there is no excluded volume for scatterers.

We next explore the off-diagonal elements of $\left\langle\tilde{O}\left(\tilde{\omega}_{1}, \tilde{\omega}_{2}\right)\right\rangle$. The average $\left\langle\tilde{O}\left(\tilde{\omega}_{1}, \tilde{\omega}_{2}\right)\right\rangle$ is shown in Fig. 3 as a function of the complex shift between two resonances $\left|\tilde{\omega}_{1}-\tilde{\omega}_{2}\right|$ normalized by $\Delta \omega$. The experimental data are also normalized by factors equal to 0.06 and 1.55 for the two ensembles, respectively. $\left\langle\tilde{O}\left(\tilde{\omega}_{1}, \tilde{\omega}_{2}\right)\right\rangle$ is seen to be negative with a magnitude which decreases with $\left|\tilde{\omega}_{1}-\tilde{\omega}_{2}\right|$ and to collapse to a single curve for the two ensembles. The magnitude of normalization factors show that the elements are stronger for strongly coupled antennas as a consequence of greater nonorthogonality. We note that it is possible in principle to have two modes which are close in central frequency, but which have very different linewidths. This would then imply that their resonance poles are distant in the complex plane and so the effects of nonorthogonality would be weak.

Chalker and Mehlig calculated the eigenvector correlator of $M \times M$ non-Hermitian random matrices of the Ginibre complex Gaussian ensemble [10,12,34],

$$
\left\langle O\left(\tilde{\omega}_{1}, \tilde{\omega}_{2}\right)\right\rangle \sim-\frac{1}{|\delta \tilde{\omega}|^{4}}\left[1-\left(1+|\delta \tilde{\omega}|^{2}\right) \exp \left(-|\delta \tilde{\omega}|^{2}\right)\right],
$$

where $\delta \tilde{\omega}=\sqrt{M}\left(\tilde{\omega}_{1}-\tilde{\omega}_{2}\right)$ is essentially the complex spacing between resonances. This result was confirmed in calculations for non-Hermitian random matrices describing the statistical properties of resonances in open chaotic cavities [11]. To compare theoretical and experimental results, the complex spacing in Eq. (4) is normalized by the average level spacing and we fit the experimental results for $\left\langle\tilde{O}\left(\tilde{\omega}_{1}, \tilde{\omega}_{2}\right)\right\rangle$ with a scale factor $a$ for the complex frequency difference of the order of unity, $|\delta \tilde{\omega}|=\left|\tilde{\omega}_{1}-\tilde{\omega}_{2}\right| /(a \Delta \omega)$, with $a=5.6$. The magnitude of $\left\langle\tilde{O}\left(\tilde{\omega}_{1}, \tilde{\omega}_{2}\right)\right\rangle$ is also normalized so that $\left\langle\tilde{O}\left(\tilde{\omega}_{1}, \tilde{\omega}_{2}\right)\right\rangle \rightarrow-1$ as $|\delta \tilde{\omega}| \rightarrow 0$. Good agreement is obtained between theory and experimental data with a power-law tail of $\left\langle\tilde{O}\left(\tilde{\omega}_{1}, \tilde{\omega}_{2}\right)\right\rangle$ as $\left|\tilde{\omega}_{1}-\tilde{\omega}_{2}\right|^{-4}$ for $\left|\tilde{\omega}_{1}-\tilde{\omega}_{2}\right|>\Delta \omega$ is confirmed in the inset. This result suggests universal average modal correlations in agreement with random matrix theory predictions for the eigenvector correlations after suitable rescaling [11].

\section{DISTRIBUTION AND SCALING OF MODAL STRENGTHS IN TRANSMISSION}

We now investigate the impact of the correlation of eigenfunctions on transport through random media. Microwave measurements in the region of moderate modal overlap have shown that modal strength in transmission may be enhanced in isolated cases and interference between modal speckle patterns tends to suppress transmission below the incoherent sum of modal contributions $[3,42,47]$.

We first analyze the variation of modal strengths in transmission, which are the diagonal elements $T_{n}=\tilde{O}_{n n}$, with the Thouless number $\delta$. The Thouless number is equal to the average of the dimensionless conductance $\delta=g$, which also falls when modes are more strongly localized and transport is suppressed [21,45]. Since $\sqrt{K_{n}} \Gamma_{n}=\left\|W_{n}\right\|^{2}, T_{n}$ can be expressed as the product of two terms of different origins:

$$
T_{n}=\frac{4\left\|W_{R n}\right\|^{2}\left\|W_{L n}\right\|^{2}}{\left(\left\|W_{R n}\right\|^{2}+\left\|W_{L n}\right\|^{2}\right)^{2}} K_{n} \equiv C_{n} K_{n} .
$$

Here, $C_{n}=\frac{4\left\|W_{R n}\right\|^{2}\left\|W_{L n}\right\|^{2}}{\left(\left\|W_{R n}\right\|^{2}+\left\|W_{L n}\right\|^{2}\right)^{2}}$, with $C_{n} \leqslant 1$, is the coupling asymmetry for the $n$th eigenfunctions between the left and right boundaries, which reflects the spatial pattern of the eigenfunctions within the sample, and $K_{n}$ is the Petermann factor.

We investigate the statistics of $T_{n}$ in random media in the crossover from diffusion to localization using the tightbinding Hamiltonian (TBH) model $[48,49]$. For strongly localized waves, the modal overlap and the conductance are small, $g \ll 1$. We find that the bulk of the distribution $P\left(T_{n}\right)$ shown in Fig. 4(a) is bimodal with peaks at $T_{n}=0$ and 

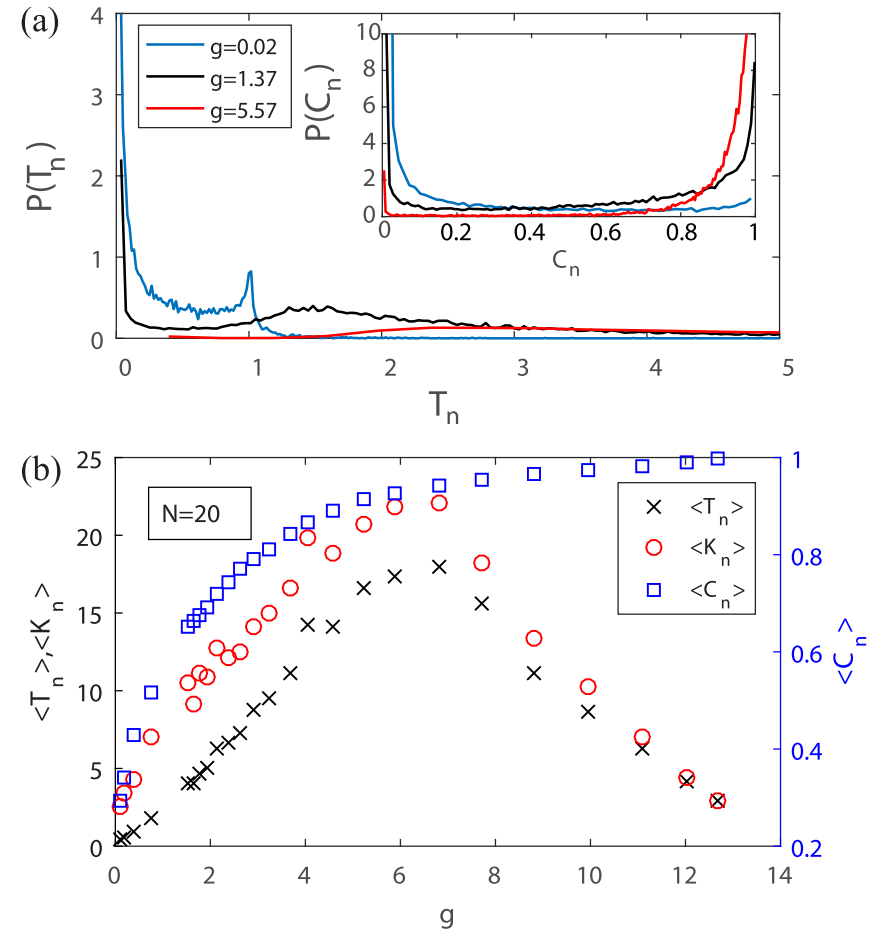

FIG. 4. (a) Simulations of $P\left(T_{n}\right)$ for samples with $g=0.02$ (blue line), $g=1.37$ (black line), and $g=5.57$ (red line). The corresponding distributions of $P\left(C_{n}\right)$ are shown in the inset. (b) Variation of $\left\langle K_{n}\right\rangle,\left\langle T_{n}\right\rangle$, and $\left\langle C_{n}\right\rangle$ with the conductance $g$.

$T_{n}=1$. This is a consequence of the bimodal distribution of the asymmetry factors $C_{n}$ of spatially localized modes. The bulk of the distribution can be explained by considering the coupling to a localized eigenstate exponentially peaked at $x_{0}$ in the sample with localization length $\xi$. The strength of the eigenfunctions at the left and right interfaces is given by $\left\|W_{L n}\right\|^{2} \sim e^{-x_{0} / \xi}$ and $\left\|W_{R n}\right\|^{2} \sim e^{-\left(L-x_{0}\right) / \xi}$. Hence, Eq. (5) gives $C_{n} \sim \cosh ^{-2}\left(\frac{L-2 x_{O}}{2 \xi}\right)$. Assuming a uniform distribution of $x_{0}$ between 0 and $L$ leads to a bimodal distribution of $C_{n}$ [50]. The bimodal distribution of $C_{n}$ and hence $T_{n}$ is in agreement with the formula proposed for isolated peaks in the transmission spectrum of one-dimensional (1D) samples using a resonator model associated with effective cavities of length $\xi[50,51]$. When transmission is dominated by a single mode, $K_{n} \sim 1$ and $T_{n} \sim C_{n}$, but modes known as necklace states may occasionally overlap spectrally and spatially even in an ensemble in which $g<1$ [52-54]. $K_{n}$ may then be large and $T_{n}$ can significantly exceed unity to produce a tail in $P\left(T_{n}\right)$.

For diffusive waves, $g>1$, the coupling to the surroundings increases and modes overlap spectrally. The eigenstates are extended throughout the sample and the coupling to the modes from the left and the right sides are typically similar so that $\left\|W_{L n}\right\|^{2} \sim\left\|W_{R n}\right\|^{2}$. Hence, $C_{n} \sim 1$ and the lower peak in $P\left(C_{n}\right)$ and $P\left(T_{n}\right)$ disappears. The probability distributions of $K_{n}$ and $T_{n} \sim K_{n}$ are then broad with peaks shifting towards values much greater than unity. We find values of $T_{n}$ as large as 150 .

The variation of $\left\langle C_{n}\right\rangle,\left\langle K_{n}\right\rangle$, and $\left\langle T_{n}\right\rangle$ with $g$ are shown in Fig. 4(b). $\left\langle K_{n}\right\rangle$ and $\left\langle T_{n}\right\rangle$ first increase with $g$ as the correlation between eigenfunctions increases, but then decrease once

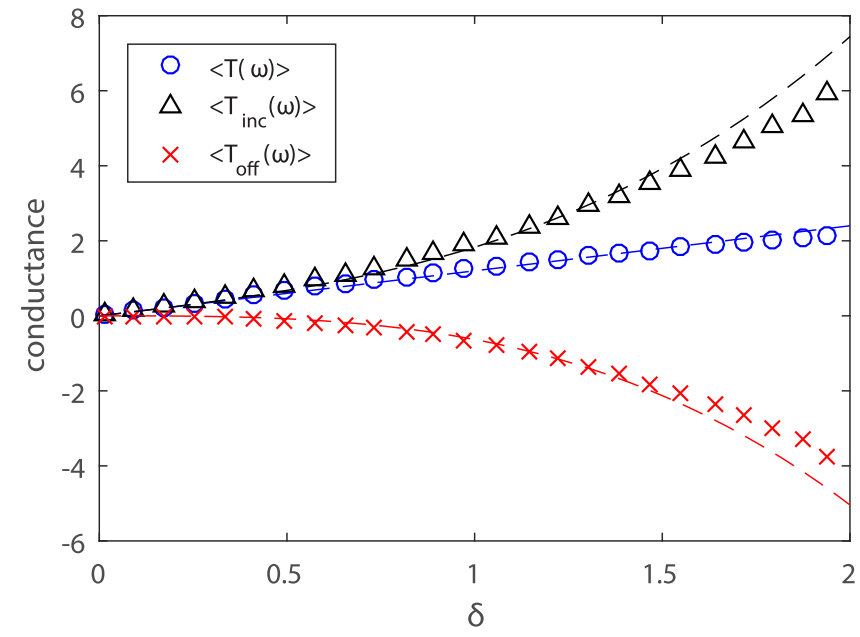

FIG. 5. Random matrix theory simulations of the scaling of the transmittance (blue circles), and the sum of the diagonal (black triangles) and off-diagonal (red crosses) modal contributions as a function of $\delta$. The dashed lines are the fits using analytical expression given in the Supplemental Material [43].

the sample is translucent, $g \geqslant N / 2$. The eigenfunctions in this regime are only slightly perturbed from the orthonormal eigenfunctions of the empty waveguide so the degree of nonorthogonality is small.

\section{SCALING OF DIAGONAL AND OFF-DIAGONAL MODAL CONTRIBUTIONS TO THE TRANSMITTANCE}

It is interesting to consider separately the contributions to the transmittance of the on- and off-diagonal terms. Using Eq. (2), the transmittance $T(\omega)$ can be expressed in terms of modal components as

$$
T(\omega)=\Sigma_{n} T_{n}(\omega)+\Sigma_{n \neq m} \frac{\left[W_{R m}^{\dagger} W_{R n}\right]\left[W_{L m}^{\dagger} W_{L n}\right]}{\left(\omega-\tilde{\omega}_{n}\right)\left(\omega-\tilde{\omega}_{m}^{*}\right)} .
$$

The impact of destructive interference of modes in transmission through diffusive media is seen in the strong suppression of transmission from the incoherent sum of transmission of $\delta \sim g$ nearly resonant modes. A perturbative approach in the limit of small modal overlap [36] shows that $\left\langle K_{n}\right\rangle \equiv\left\langle O_{n n}\right\rangle$ increases as $\sim 1+\delta^{2}$ (see Supplemental Material [43]). $\delta$ modes contribute to transmission so that the incoherent sum of modal strengths $\left\langle T_{\text {inc }}(\omega)\right\rangle=\left\langle\Sigma_{n} T_{n}(\omega)\right\rangle$ is increased relative to $g=\langle T(\omega)\rangle$ by a term scaling as $\delta^{3} \sim g^{3}$. Transmission is then reduced by destructive interference between correlated modal components with $n \neq m$ in Eq. (6) ensuring that transmission is bounded by unity. The contribution of off-diagonal terms to the average transmittance scales as $-\delta^{3}$, as expected to satisfy $g \sim \delta[43]$.

Statistics of modes in chaotic cavities are well described by random matrix theory (RMT). The $M \times M$ internal Hamiltonian $H_{0}$ of the effective Hamiltonian $H_{\text {eff }}=H_{0}-\frac{i}{2} \gamma V V^{T}$ is modeled by a real symmetric matrix drawn from the Gaussian orthogonal ensemble with $\left\langle\left(H_{0}\right)_{i j}^{2}\right\rangle=1 / M$. The coupling matrix $V$ is a real random matrix with Gaussian distribution and $\left\langle V_{i j} V_{k l}\right\rangle=\delta_{i j} \delta_{k l} / M$. The modal overlap increases with increasing coupling strength of the channels to the system, 
$\kappa=\gamma / 2$. The coupling strength to the continuum is $\kappa_{c}=$ $\pi \gamma /(2 M D)$, where $D=\pi / M$ is the mean level spacing at the center of the band, $E=0$. The coupling strength is related to the transmission coefficient through the channels, $T_{c}$, with $T_{c}=4 \kappa /(1+\kappa)^{2}$. The scattering matrix $S=1-$ $i V^{T}\left[\omega-H_{\mathrm{eff}}\right]^{-1} V$ gives reflection and transmission coefficients through the medium. Numerical results of the conductance found from these RMT simulations shown in Fig. 5 are in excellent agreement with the perturbative approach for $\delta<1.4$.

\section{CONCLUSION}

Modal correlation provides a fresh vantage point from which to understand scattering from, transmission through, or energy disposition within nuclei, large molecules, disordered photonic and plasmonic systems, chaotic cavities, and multimode fibers [4,6,55-58]. Surprisingly, we find that the strength of correlation of eigenfunctions increases with the number of resonant modes participating in transmission, $g$, while the degree of spatial and spectral intensity, which is approximately $1 / g$ in diffusive media, falls with the number of modes in resonance. This is a result of the balance between positive and negative correlation found in the diagonal and off-diagonal elements of the transmission overlap matrix. Since the TM can be expressed in terms of MTMs, the ability to approach perfect transmission in diffusive systems and the maximal contrast in focusing through random systems [56,58] have their origin in the correlation exhibited in the transmission overlap matrix and increases with the number of resonant modes participating in transmission. The modal basis of the TM is also important in the control of transmission and delay times of broadband pulses $[47,59,60]$ since, in contrast to the MTMs, the TM is defined at a single frequency.

\section{ACKNOWLEDGMENTS}

This publication was supported by the European Union through the European Regional Development Fund (ERDF), by the French region of Brittany and Rennes Métropole through the CPER Project SOPHIE/STIC \& Ondes, and by the National Science Foundation under Grant No. DMR/BSF: 1609218. We would like to acknowledge Jing Wang for discussions of early measurements of modal correlation and Zhou Shi, Ulrich Kühl, Olivier Legrand, and Fabrice Mortessagne for stimulating discussions.
[1] E. S. C. Ching, P. T. Leung, A. Maassen van den Brink, W. M. Suen, S. S. Tong, and K. Young, Quasinormal-mode expansion for waves in open systems, Rev. Mod. Phys. 70, 1545 (1998).

[2] U. Kuhl, R. Höhmann, J. Main, and H. J. Stöckmann, Resonance Widths in Open Microwave Cavities Studied by Harmonic Inversion, Phys. Rev. Lett. 100, 254101 (2008).

[3] J. Wang and A. Z. Genack, Transport through modes in random media, Nature 471, 345 (2011).

[4] C. Sauvan, J. P. Hugonin, I. S. Maksymov, and P. Lalanne, Theory of the Spontaneous Optical Emission of Nanosize Photonic and Plasmon Resonators, Phys. Rev. Lett. 110, 237401 (2013).

[5] F. Alpeggiani, N. Parappurath, E. Verhagen, and L. Kuipers, Quasinormal-Mode Expansion of the Scattering Matrix, Phys. Rev. X 7, 021035 (2017).

[6] P. Lalanne, W. Yan, K. Vynck, C. Sauvan, and J.-P. Hugonin, Light interaction with photonic and plasmonic resonances, Laser Photonics Rev. 12, 1700113 (2018).

[7] A. Asenjo-Garcia, J. D. Hood, D. E. Chang, and H. J. Kimble, Atom-light interactions in quasi-one-dimensional nanostructures: A Green's-function perspective, Phys. Rev. A 95, 033818 (2017).

[8] P. T. Leung, W. M. Suen, C. P. Sun, and K. Young, Waves in open systems via a biorthogonal basis, Phys. Rev. E 57, 6101 (1998).

[9] I. Rotter, A non-Hermitian Hamilton operator and the physics of open quantum systems, J. Phys. A 42, 153001 (2009).

[10] J. T. Chalker and B. Mehlig, Eigenvector Statistics in NonHermitian Random Matrix Ensembles, Phys. Rev. Lett. 81, 3367 (1998).

[11] B. Mehlig and M. Santer, Universal eigenvector statistics in a quantum scattering ensemble, Phys. Rev. E 63, 020105(R) (2001).
[12] Y. V. Fyodorov, On statistics of bi-orthogonal eigenvectors in real and complex Ginibre ensembles: Combining partial Schur decomposition with supersymmetry, Commun. Math. Phys. 363, 579 (2018).

[13] E. P. Wigner, On the statistical distribution of the widths and spacings of nuclear resonance levels, Math. Proc. Cambridge Philos. Soc. 47, 790 (1951).

[14] C. Porter and R. Thomas, Fluctuations of nuclear reaction widths, Phys. Rev. 104, 483 (1956).

[15] F. J. Dyson, Statistical theory of the energy levels of complex systems, J. Math. Phys. 3, 140 (1962).

[16] I. Rotter, A continuum shell model for the open quantum mechanical nuclear system, Rep. Prog. Phys. 54, 635 (1991).

[17] G. E. Mitchell, A. Richter, and H. A. Weidenmüller, Random matrices and chaos in nuclear physics: Nuclear reactions, Rev. Mod. Phys. 82, 2845 (2010).

[18] E. Abrahams, P. W. Anderson, D. C. Licciardello, and T. V. Ramakrishnan, Scaling Theory of Localization: Absence of Quantum Diffusion in Two Dimensions, Phys. Rev. Lett. 42, 673 (1979).

[19] Y. Imry and R. Landauer, Conductance viewed as transmission, Rev. Mod. Phys. 71, S306 (1999).

[20] A. Z. Genack, Optical Transmission in Disordered Media, Phys. Rev. Lett. 58, 2043 (1987).

[21] A. D. Mirlin, Statistics of energy levels and eigenfunctions in disordered systems, Phys. Rep. 326, 259 (2000).

[22] T. Kottos, Statistics of resonances and delay times in random media: Beyond random matrix theory, J. Phys. A: Math. Gen. 38, 10761 (2005).

[23] A. Beige, D. Braun, B. Tregenna, and P. L. Knight, Quantum Computing Using Dissipation to Remain in a Decoherence-Free Subspace, Phys. Rev. Lett. 85, 1762 (2000). 
[24] R. El-Ganainy, K. G. Makris, M. Khajavikhan, Z. H. Musslimani, S. Rotter, and D. N. Christodoulides, NonHermitian physics and PT symmetry, Nat. Phys. 14, 11 (2018).

[25] M.-A. Miri and A. Alù, Exceptional points in optics and photonics, Science 363, eaar7709 (2019).

[26] K. Petermann, Calculated spontaneous emission factor for double-heterostructure injection lasers with gain-induced waveguiding, IEEE J. Quantum Electron. 15, 566 (1979).

[27] Y.-J. Cheng, C. G. Fanning, and A. E. Siegman, Experimental Observation of a Large Excess Quantum Noise Factor in the Linewidth of a Laser Oscillator Having Nonorthogonal Modes, Phys. Rev. Lett. 77, 627 (1996).

[28] K. Frahm, H. Schomerus, M. Patra, and C. Beenakker, Large Petermann factor in chaotic cavities with many scattering channels, Europhys. Lett. 49, 48 (2000).

[29] H. Lourenço-Martins, P. Das, L. H. Tizei, R. Weil, and M. Kociak, Self-hybridization within non-Hermitian localized plasmonic systems, Nat. Phys. 14, 360 (2018).

[30] H. E. Türeci, H. G. L. Schwefel, P. Jacquod, and A. D. Stone, Modes of wave-chaotic dielectric resonators, in Progress in Optics, Vol. 47, edited by E. Wolf (Elsevier, Amsterdam, 2005), Chap. 2, pp. 75-137.

[31] H. Cao and J. Wiersig, Dielectric microcavities: Model systems for wave chaos and non-Hermitian physics, Rev. Mod. Phys. 87, 61 (2015).

[32] Y. V. Fyodorov and D. V. Savin, Statistics of Resonance Width Shifts as a Signature of Eigenfunction Nonorthogonality, Phys. Rev. Lett. 108, 184101 (2012).

[33] J. B. Gros, U. Kuhl, O. Legrand, F. Mortessagne, E. Richalot, and D. V. Savin, Experimental Width Shift Distribution: A Test of Nonorthogonality for Local and Global Perturbations, Phys. Rev. Lett. 113, 224101 (2014).

[34] R. A. Janik, W. Nörenberg, M. A. Nowak, G. Papp, and I. Zahed, Correlations of eigenvectors for non-Hermitian randommatrix models, Phys. Rev. E 60, 2699 (1999).

[35] P. T. Leung, S. Y. Liu, and K. Young, Completeness and orthogonality of quasinormal modes in leaky optical cavities, Phys. Rev. A 49, 3057 (1994).

[36] C. Poli, D. V. Savin, O. Legrand, and F. Mortessagne, Statistics of resonance states in open chaotic systems: A perturbative approach, Phys. Rev. E 80, 046203 (2009).

[37] O. Xeridat, C. Poli, O. Legrand, F. Mortessagne, and P. Sebbah, Quasimodes of a chaotic elastic cavity with increasing local losses, Phys. Rev. E 80, 035201(R) (2009).

[38] C. Poli, O. Legrand, and F. Mortessagne, Statistics of resonance states in a weakly open chaotic cavity with continuously distributed losses, Phys. Rev. E 82, 055201(R) (2010).

[39] E. N. Bulgakov, I. Rotter, and A. F. Sadreev, Correlated behavior of conductance and phase rigidity in the transition from the weak-coupling to the strong-coupling regime, Phys. Rev. B 76, 214302 (2007).

[40] Y. H. Kim, U. Kuhl, H. J. Stöckmann, and P. W. Brouwer, Measurement of Long-Range Wave-Function Correlations in an Open Microwave Billiard, Phys. Rev. Lett. 94, 036804 (2005).

[41] W. A. Hamel and J. P. Woerdman, Observation of Enhanced Fundamental Linewidth of a Laser due to Nonorthogonality of its Longitudinal Eigenmodes, Phys. Rev. Lett. 64, 1506 (1990).
[42] M. Davy and A. Z. Genack, Selectively exciting quasi-normal modes in open disordered systems, Nat. Commun. 9, 4714 (2018).

[43] See Supplemental Material at http://link.aps.org/supplemental/ 10.1103/PhysRevResearch.1.033026 for derivation of Eq. (2), details on the Harmonic inversion technique, additional simulation results, and considerations on the scaling of modal strengths.

[44] A. Goetschy and A. D. Stone, Filtering Random Matrices: The Effect of Incomplete Channel Control in Multiple Scattering, Phys. Rev. Lett. 111, 063901 (2013).

[45] D. J. Thouless, Maximum Metallic Resistance in Thin Wires, Phys. Rev. Lett. 39, 1167 (1977).

[46] R. Landauer, Electrical resistance of disordered one-dimensional lattices, Philos. Mag. 21, 863 (1970).

[47] Z. Shi and A. Z. Genack, Dynamic and spectral properties of transmission eigenchannels in random media, Phys. Rev. B 92 , 184202 (2015).

[48] T. Kottos and M. Weiss, Statistics of Resonances and Delay Times: A Criterion for Metal-Insulator Transitions, Phys. Rev. Lett. 89, 056401 (2002).

[49] G. L. Celardo, A. M. Smith, S. Sorathia, V. G. Zelevinsky, R. A. Sen'kov, and L. Kaplan, Transport through nanostructures with asymmetric coupling to the leads, Phys. Rev. B 82, 165437 (2010).

[50] L. Zhao, C. Tian, Y. P. Bliokh, and V. Freilikher, Controlling transmission eigenchannels in random media by edge reflection, Phys. Rev. B 92, 094203 (2015).

[51] Z. Q. Zhang, A. A. Chabanov, S. K. Cheung, C. H. Wong, and A. Z. Genack, Dynamics of localized waves: Pulsed microwave transmissions in quasi-one-dimensional media, Phys. Rev. B 79, 144203 (2009).

[52] J. Pendry, Quasi-extended electron states in strongly disordered systems, J. Phys. C 20, 733 (1987).

[53] J. Bertolotti, S. Gottardo, D. S. Wiersma, M. Ghulinyan, and L. Pavesi, Optical Necklace States in Anderson Localized 1D Systems, Phys. Rev. Lett. 94, 113903 (2005).

[54] K. Y. Bliokh, Y. P. Bliokh, V. Freilikher, A. Z. Genack, and P. Sebbah, Coupling and Level Repulsion in the Localized Regime: From Isolated to Quasiextended Modes, Phys. Rev. Lett. 101, 133901 (2008).

[55] P. T. Kristensen and S. Hughes, Modes and mode volumes of leaky optical cavities and plasmonic nanoresonators, ACS Photonics 1, 2 (2014).

[56] A. P. Mosk, A. Lagendijk, G. Lerosey, and M. Fink, Controlling waves in space and time for imaging and focusing in complex media, Nat. Photonics 6, 283 (2012).

[57] S. Rotter and S. Gigan, Light fields in complex media: Mesoscopic scattering meets wave control, Rev. Mod. Phys. 89, 015005 (2017).

[58] C. W. Hsu, S. F. Liew, A. Goetschy, H. Cao, and A. D. Stone, Correlation-enhanced control of wave focusing in disordered media, Nat. Phys. 13, 497 (2017).

[59] C. W. Hsu, A. Goetschy, Y. Bromberg, A. D. Stone, and H. Cao, Broadband Coherent Enhancement of Transmission and Absorption in Disordered Media, Phys. Rev. Lett. 115, 223901 (2015).

[60] P. Ambichl, W. Xiong, Y. Bromberg, B. Redding, H. Cao, and S. Rotter, Super- and Anti-Principal-Modes in Multimode Waveguides, Phys. Rev. X 7, 041053 (2017). 